\title{
Deaf ACCESS: Adapting Consent Through Community Engagement and State-of-the-Art Simulation
}

\author{
Melissa L. Anderson* ${ }^{*}$, , Timothy Riker ${ }^{\ddagger}$, Stephanie Hakulin, Jonah Meehan, \\ Kurt Gagne, Todd Higgins, Elizabeth Stout, Emma Pici-D’Ottavio, \\ Kelsey Cappetta ${ }^{\#}$ and Kelly S. Wolf Craig
}

\author{
University of Massachusetts Medical School \\ ${ }^{*}$ Correspondence should be sent to Melissa L. Anderson, Implementation Science \& Practice Advances Research Center (iSPARC), University of \\ Massachusetts Medical School (UMMS), 222 Maple Avenue, Chang Building, Shrewsbury, MA 01545 (e-mail: melissa.anderson@umassmed.edu)
}

\begin{abstract}
One of the most understudied health disparity populations in the United States is the Deaf community, a sociolinguistic minority group of more than 500,000 individuals who communicate via American Sign Language. Research on Deaf health disparities is lacking due to inaccessible recruitment, sampling, and data collection procedures, as well as the fundamental disconnect between medical and cultural views of Deaf people. A potential starting place for addressing inaccessible research methods and mistrust of the biomedical research community is the careful reconsideration of the traditional informed consent process, often a Deaf individual's first point of contact with the research world. Yet, most Deaf individuals experience obstacles to engaging in informed consent due to differences in language and development compared to hearing individuals. In response to these issues, our team led a three-phase, formative, community-engaged approach to adapt the informed consent process and train research staff in the updated method so that all required components are properly communicated and understood. The goals of our work were to promote Deaf engagement in research about the Deaf community, increase the number of Deaf individuals who participate in general population biomedical research, and generalize our findings to improve research accessibility for the general population.
\end{abstract}

Recent research employing an American Sign Language (ASL)accessible public health survey identified significant health disparities experienced by the U.S. Deaf community (Barnett, Klein, et al., 2011), a sociolinguistic minority group of more than 500,000 Americans who communicate via ASL (Mitchell, Young, Bachleda, \& Karchmer, 2006). Compared to the general population, Deaf individuals are more likely to be obese, to have attempted suicide in the past year, and to have experienced physical abuse and forced sex (Barnett, Klein, et al., 2011). These findings are unique in that they arose from the first national endeavor to collect health data from the Deaf population in ASL. The use of other national public health data to determine Deaf health priorities have historically been obstructed by barriers described below.

\section{Barriers to Deaf Research Participation}

Further research on Deaf health disparities is lacking due, in part, to recruitment, sampling, and data collection procedures that are inaccessible to Deaf people (Barnett, McKee, Smith, \& Pearson, 2011; Fellinger, Holzinger, \& Pollard, 2012; Livermore, Whalen, Prenovitz, Aggarwal, \& Bardos, 2011). Random-digit-dial surveys fail to sample Deaf ASL users, who use videophones rather than standard telephone technology. In-person studies,

\footnotetext{
$\dagger$, http://orcid.org/0000-0001-6141-5013

f Timothy Riker is also affiliated with the Center for Language Studies, Brown University.

\# Kelsey Cappetta is also affiliated with the Department of Psychology, Gallaudet University.

Received June 12, 2019; revisions received July 31, 2019; accepted July 31, 2019
}

(c) The Author(s) 2019. Published by Oxford University Press. All rights reserved. For Permissions, please email: journals.permissions@oup.com 
such as the National Comorbidity Study Replication, often sample English users only and do not document any use of accommodations for Deaf individuals (Anderson, Ziedonis, \& Najavits, 2014).

The lack of research within this population is exacerbated by a fundamental disconnect between biomedical and cultural views of Deaf people (McKee, Schlehofer, \& Thew, 2013). Researchers generally follow the "medical model" of deafness, focusing on how to "cure" or "fix" hearing loss (Bauman, 2004; Ladd, 2003; Lane, 1992). Most Deaf community members, however, do not believe they need to be "fixed," but that they are members of a minority group with rich culture, shared experience, history, art, and literature (Bauman, 2004; Ladd, 2003; Lane, 1992). This disconnect has fueled a long history of mistreatment against Deaf people in the research world. Common missteps have included failure to provide ASL interpreters for participation in research studies, failure to explain research procedures and obtain consent in Deaf participants' primary language, and an overwhelming focus on research questions meant to "solve the problem of deafness" (Lane, 2005; McKee et al., 2013). More egregious biomedical abuses include the use of eugenics and sterilization to prevent the growth of the Deaf community (Lane, 2005; McKee et al., 2013) and underlie a communal feeling of mistrust toward researchers (McKee et al., 2013).

\section{Reconsidering Traditional Informed Consent Procedures}

A potential starting place for addressing inaccessible research methods and mistrust of the biomedical research community is the careful reconsideration of the traditional informed consent process, which is often a Deaf individual's first point of contact with the research world. Most Deaf individuals experience obstacles to engaging in informed consent due to differences in language and development compared to hearing individuals (Glickman, 2013).

Often, Deaf people's first natural language is ASL and written English is acquired as a second language; this means that English literacy varies widely among Deaf people, with research suggesting a fourth-grade median English reading level among Deaf high school graduates (Morere, 2011). Low health literacy is also common due to limited language access during key developmental periods and "a lifetime of limited access to information that is often considered common knowledge among hearing persons" (Barnett, McKee, et al., 2011). Health-related vocabulary among Deaf sign language users parallels non-English-speaking U.S. immigrants (McEwen \& Anton-Culver, 1988), and "many adults deaf since birth or early childhood do not know their own family medical history, having never overheard their hearing parents discussing this with their doctor" (Barnett, McKee, et al., 2011).

Yet, most informed consent protocols rely on written English consent forms, which include jargon and legal terms that are inaccessible to the common layperson, regardless of hearing status. Such methods fail to produce a fully informed Deaf research participant and call for adaptations aboveand-beyond simple translation of informed consent materials into ASL.

\section{Study Objectives}

In response to these issues, our team led Deaf ACCESS: Adapting Consent through Community Engagement and State-of-the-art
Simulation, a three-phase, formative, community-engaged approach to adapt the informed consent process and train research staff in the updated method so that all required components are properly communicated and understood. The goals of our work were to promote Deaf engagement in research about the Deaf community, increase the number of Deaf individuals who participate in general population biomedical research, and generalize our findings to improve research accessibility for members of the general population.

Our study objectives drew on the pilot work of Jeroan Allison, MD, MS-the Simulation-based Community-engaged Research Intervention for Informed Consent Protocol Testing and Training (SCRIIPTT). The goal of SCRIIPTT was to incorporate culturally and linguistically competent methods into the informed consent process by leveraging the expertise of the Massachusetts African-American and Latino communities. Formative assessment identified barriers and facilitators to community engagement in research, and this knowledge informed the prototype development of an experiential training intervention for research staff. Throughout this process, the local community served by the University of Massachusetts Medical School participated as an equal partner-the critical factor in the successes of both SCRIIPTT and Deaf ACCESS.

\section{Formation of a Community-Engaged Research Team}

To conduct community-engaged data collection, analysis, and dissemination, our first effort was to cultivate a Deaf-majority research team. Six out of eight of our team members were Deaf; seven of eight were fluent in ASL.

Our principal investigator is a hearing psychologist who conducts clinical research within the Deaf community. As a hearing individual conducting Deaf-engaged research, she believed that her primary role was to provide administrative guidance and consultation on scientific methodology throughout the study. She co-led the team with a Deaf co-investigator, a university lecturer of Language Studies. Their collaboration began prior to the initiation of any research procedures-during the grant writing phase-to ensure that the proposed research activities would align with the priorities of the Deaf community. This collaborative leadership team was supported by another Deaf coinvestigator, as well as a hearing research coordinator.

The remainder of our research team was comprised of four Deaf Community Advisors-laypersons from the Deaf community who became active members of the research team. The primary role of our Deaf Community Advisors was to guide the design of study methodology in such a way as to represent the diverse experiences, perspectives, and needs of the Deaf community. To achieve this goal, we attracted a diverse team of Deaf community members to these positions via job postings in both ASL video and written English formats. We started with a set of essential job qualifications-ASL fluency, expertise in Deaf cultural norms, awareness of access barriers, and ability to use a computer. Beyond these qualifications, we were mindful of creating a team diverse with respect to race/ethnicity, gender, sexual orientation, level of education, educational background (i.e., Deaf residential school, mainstream school), and socioeconomic status. In addition to these diversity factors, our selection process was also attuned to how each Deaf Community Advisor contributed a complementary skill set to the team-in our case, filmmaking, scriptwriting, community outreach and networking, acting, and writing. 
Similar to other community-engaged research teams, some of our Deaf Community Advisors had prior experience in research settings, while others had none. In this situation, "the researchers work with community members to develop a capacity-building strategy in which community members receive training on research theories and methodology; in turn, these members contribute to the project by taking on research activities such as recruitment of participants, interviews, translation, and data analysis" (Wong \& Poon, 2010). This approach directly applies to the complementary expertise and the cross-training experiences that occurred within our research team. The principal investigator provided formal training and guidance regarding research ethics, strict procedures for protecting privacy and maintaining confidentiality in a small community context, study methodology, and studyspecific content knowledge. The co-investigators and Deaf Community Advisors provided linguistic and cultural expertise for development of appropriate study methodology, conduct of analysis and interpretation of data, and dissemination of findings back to the community. When issues arose due to incongruences between Deaf cultural norms and traditional empirical approaches, our team collaboratively decided how to manage these issues. This ongoing dialogue informed our approaches to data collection, data analysis/interpretation, and dissemination of findings, as described below.

\section{Phase 1: Community Forums}

During the first phase of the Deaf ACCESS study, we sought to identify barriers and facilitators to the full engagement of the Deaf community in biomedical research, with the goal of informing later stages of our project (i.e., design of focus groups, development of researcher training intervention). We began this process by first deepening the dialogue between the Deaf community and University of Massachusetts Medical School researchers via three community forums hosted at local Deaf community cultural institutions across New England (e.g., Deaf residential schools, Deaf advocacy agencies) between October and November 2016. The Truth and Reconciliation Model (Twombley, 2014) guided the structure of each forum, described below.

\section{Community Forum Methods}

Recruitment. Community forum participants were recruited via postings to Deaf listservs, Deaf Facebook groups, and Deaffocused organizations. Recruitment materials (i.e., ASL vlogs and plain English flyer) were designed in collaboration with our Deaf Community Advisors to more effectively engage members of the Deaf community. As the forums were intended for anyone who identified as a member of the Deaf community, inclusion criteria were minimal: (a) age 18 and above; (b) self-identification as a member of the Deaf community; and (c) willingness and ability to attend a community forum at one of three community locations. The only exclusion criterion was inability to provide consent to participate due to guardianship.

Ethics. Each of the three community forums began with an onsite consent process, in which the principal investigator and coinvestigator presented the study fact sheet in ASL. The community forums qualified for a waiver of written documentation of consent, as they were considered minimal risk and involved no procedures for which written consent is normally required outside of the research context. A written copy of the fact sheet was provided to each participant, and time was given to review the fact sheet and ask any clarifying questions.

Procedures. Each community forum was hosted in a Deaf community location, was designated as a "Deaf Space" (i.e., a Deafonly space where forum members could feel safe sharing their experiences and perspectives in an uninhibited manner), and was co-led by a Deaf facilitator. The planning, structure, and function of each forum were guided by the Truth and Reconciliation Model (Twombley, 2014), with Deaf community members and hearing researchers first holding an open conversation about Deaf people's history of mistreatment in the research world and then collaboratively exploring steps needed to move forward. Participants were free to share their stories about experiences in the research world and to make recommendations about how researchers can better include Deaf people in their studies. In addition to the hearing principal investigator, one or two hearing researchers from the University of Massachusetts Medical School and other academic institutions were present at each forum to serve as representatives of the research community and to issue a formal apology on behalf of this community.

The principal investigator and co-investigator co-led the forum discussion in ASL. Seating was arranged in a semicircle format to provide full visual access to attendees. Three ASL interpreters were hired to facilitate communication between signing and non-signing attendees. Five Certified Deaf Interpreters (CDIs) were also present to provide specialized support to participants with varying language backgrounds and communication needs. (Note: A CDI is a Deaf individual who demonstrates knowledge and understanding of interpreting, the Deaf community and culture, training/experience in use of gesture, mime, props, drawings, and other tools to enhance communication.) The interpreting team worked together to ensure that all participants received the same information and had equal opportunity to participate in the discussion. Forum discussion (in both ASL and spoken English) was recorded via five video cameras surrounding the room, simultaneously recording each sector of the semicircle.

Measures. Forum discussion prompts were developed in collaboration with our team's Deaf Community Advisors: (a) Poll: "How many of you have seen ads for research? (in the train/bus, posters, email blasts, etc.)" [raise hands], Discussion Question: "Did you participate? Why or why not? What stopped you?"; (b) Poll: "How many of you have participated in a research study before?" [raise hands], Discussion Question: "What did you like about this/these experience(s)? What did you not like?"; (c) Discussion Question: “We have these hearing researchers here. What would you tell them to do to improve their work? What ideas/suggestions do you have for them?"; and (d) Discussion Question: "How should researchers determine Deaf community research priorities for their research questions?"

In addition to data generated from these discussion prompts, at the end of each forum, participants were asked to complete a brief, anonymous written evaluation. Evaluation questions captured basic demographic information and assessed participants' views on the impact of the apology, acknowledgement of past injustices, and their likelihood of participating in research or supporting other Deaf people to participate in research. The evaluation was distributed in plain English form, a video was displayed that guided participants through the evaluation in ASL, and CDI assistance could be requested if anyone required additional sign support.

Analyses. Videos of community forum discussions were uploaded into ATLAS.ti, where ASL responses were directly 
coded in their original form without the need for translation and transcription into written English (Anderson et al., 2018). Qualitative data were analyzed based on a grounded theory approach using two major techniques: (a) content analysis, in which the number of similar responses to questions were tallied and described and (b) a summary of the answers to the questions outlined by Casey (Krueger, 1998). Such questions include What are the participants saying? What are they feeling? What is really important? What are the themes? Are there any comments said only once but deserve to be noted? Which quotes really give the essence of the conversation? What ideas will be especially useful for intervention? Data were coded collaboratively and simultaneously by all members of the research team, with conflicting points of view further discussed and resolved among the team. For an indepth discussion of our team's linguistically and socio-politically appropriate methodology, see Anderson et al. (2018).

\section{Community Forum Results}

Twenty-two individuals completed the end-of-forum evaluation (13 female, 9 male). Mean age was 55 years old. Eighty-six percent of the sample identified as White non-Hispanic/Latino, $5 \%$ identified as Asian, and the remainder declined to report their race. Ninety-one percent identified as Deaf, 5\% as Hard of Hearing, and $9 \%$ as "other" hearing status. Eighty-two percent of participants noted ASL as their preferred language, as compared to English (23\%) and "other" languages (9\%). (Note: Where percentages exceed $100 \%$, participants were permitted to select all that applied.)

Qualitative analyses of forum video revealed the following themes: (a) "Research in the general public is not for me," that is, the assumption that communication access will not be provided; (b) General mistrust of hearing people due to history of negative experiences and oppression; (c) Failure of researchers to communicate study results back to the Deaf community; and (d) Tendency of researchers to benefit from data provided by Deaf research participants, without any efforts to "give back" to the community. One participant poignantly stated: How will your research help us? It won't! We've been trying so hard and struggling for such a long time... It will help your job and you will get paid, but how will that help us? You want us to share our experiences and use it to your personal benefit, but will not help us at all!

Quantitative evaluation results showed that $86 \%$ of participants enjoyed the opportunity to advise researchers about how to improve their work, $73 \%$ reported feeling positive about sharing their research experiences, and 59\% appreciated the apology from hearing researchers.

Despite these promising results, the number of community forum attendees $(n=22)$ was significantly lower than originally anticipated. Some attendees appeared uncomfortable sharing in such a public space and expressed that they would have preferred to have the opportunity to discuss these issues in a smaller, more intimate setting. We suspect that this sense of mistrust and fear led others to avoid attending the forums altogether. Over the course of the community forums, our team also observed that many attendees did not possess a basic understanding of the meaning of "research" and instead attended the forums in order to express their frustration with barriers and discrimination encountered within medical, vocational, and educational settings.

After analyzing the qualitative data from the first three forums, we reached saturation, with no new themes arising. Paired with the behavioral observations noted above, our team made a data-informed decision to refrain from hosting a fourth community forum during the activities of Phase 1 and instead conduct one additional focus group during Phase 2 of our study.

\section{Phase 2: Focus Groups}

During the second phase of the Deaf ACCESS study, we aimed to gain further insight into the Deaf community's knowledge of, attitudes toward, and experiences with the research informed consent process by conducting four focus groups with Deaf adults across New England between May 2017 and June 2017. The primary goal of these focus groups was to inform the final phase of our project-development of the researcher training intervention.

\section{Focus Group Methods}

Recruitment. We planned a total of four focus groups, inviting six Deaf community members to each, for a maximum of 24 participants altogether. Inclusion and exclusion criteria were again minimal and mirrored the criteria for Phase 1 community forums. Our primary recruitment effort was to contact individuals who had attended our Deaf community forums and completed contact information slips stating their interest in participating in future research.

Additionally, we hosted one focus group that was open only to CDIs, many of whom had interpreted for the community forums in Phase 1, following their special request for a space to share their experiences and perspectives. Specifically, after the community forums were complete, many of the CDIs who had facilitated communication during the forums expressed a desire to share their research experiences as well, yet, their Code of Professional Conduct restricted them from stepping out of the interpreter role and stepping into a participant role during the forums. Many approached the research team to ask if they could call us to share their experiences interacting with the research world, or if they could submit video testimonials to our team about their experiences. Ultimately, we decided that the most appropriate solution would be to hold a CDI-only focus group during Phase 2 of the study.

Ethics. Our focus groups qualified for a waiver of written documentation of consent. The fact sheet was explained in ASL by the focus group co-facilitators (the principal investigator and coinvestigator) at the beginning of each focus group. Focus group participants were informed that they had the right to refrain from answering any discussion questions posed in the focus group or any written questions posed on the post-focus group evaluation. A written copy of the fact sheet was distributed to participants, and ample time was given for the participants to review the fact sheet and ask any clarifying questions before focus group discussion began.

Procedures and measures. Each 2-hr focus group was coled by the principal investigator and the Deaf co-investigator, where all individuals in the room were fluent in ASL and no ASL interpreters were present. Focus groups were recorded via three video cameras surrounding the room, recording the co-facilitators and participants.

The structure of each focus group was guided by themes that emerged from the three community forums, as well as the expertise of our Deaf team members:

1. Explain the overall purpose of informed consent and show ASL video about the "Principles of Informed Consent": https://www.youtube.com/watch?v=ZR2G0gbfqXI 
2. Role play an example of the traditional informed consent process between a hearing participant and hearing research staff member

3. Discussion questions:

a. "Do you think this process would work for Deaf people?" "Why or why not?"

b. "What changes would you make to this process to make it more Deaf friendly?"

4. Show a typical model for a biomedical informed consent form (obtained from a pharmaceutical company's website)

5. Discussion questions:

a. "Do you think this form would work for Deaf people?" "Why or why not?"

b. "What changes would you make to make this more Deaf friendly?"

At the end of each focus group session, participants were asked to complete a brief, anonymous written evaluation that captured demographic information and assessed their views on how the concept of informed consent in research within the Deaf community was addressed. Focus group participants each received a $\$ 50$ American Express gift card for their participation.

Analyses. Video footage of the focus groups was uploaded into ATLAS.ti for qualitative analysis. Similar to analysis of community forum data described above, focus group data were analyzed based on a grounded theory approach, especially focused on identifying themes that would be useful for the development of our researcher training intervention. The research team collaboratively coded all qualitative data, with any conflicting findings discussed and resolved concurrently among the research team.

\section{Focus Group Results}

Of the 24 individuals originally scheduled for the focus groups, 18 were able to attend ( 11 female, 7 male). Eleven percent were aged 25-34 years, $28 \%$ were aged $35-44$ years old, and $61 \%$ were aged 45-64 years old. Eighty-nine percent of the sample identified as White non-Hispanic/Latino, one participant identified as White Hispanic/Latino, and one identified as Black. Fifty-six percent identified as straight, $22 \%$ as lesbian, $11 \%$ as gay, $6 \%$ as bisexual, and $6 \%$ as other. Twenty-two percent had a high school diploma, $33 \%$ a 4 -year college degree, $39 \%$ a graduate degree, and $6 \%$ a doctoral degree.

Data yielded five main themes: (a) need for system change; (b) information for researcher training intervention; (c) information regarding informed consent forms; (d) information for interpreter training intervention; and (e) other general information regarding Deaf-focused research. The structure of resulting themes and subthemes are outlined in Table 1.

\section{Phase 3: Intervention Development and Pilot Test}

"Sign Here: How to Conduct Informed Consent with Deaf Research Participants"

The themes and subthemes generated from the focus groups described above directly guided the development of our researcher training intervention, titled Sign Here: How to Conduct Informed Consent with Deaf Research Participants. Our intended target audience was hearing researchers and research staff who have had little-to-no experience interacting with Deaf people and who would be conducting informed consent via an ASL interpreter. Focus group results suggested that the ideal intervention format for this audience would be a video that spotlighted Deaf people and the use of ASL, incorporated both auditory and visual learning strategies, and leveraged humor and entertainment value to increase viewer engagement.

Our team first developed an outline of content to be included in the video script based on the identified themes from Phase 1 community forums, Phase 2 focus groups, and the expertise of Deaf team members. The team then worked together to expand this outline into a first draft of a script. One of our Deaf Community Advisors has a degree in screenwriting and had prior experience writing scripts for several ASL films.

A final draft of the script was shared with the filmmaker that we recruited to the project-an award-winning filmmaker who is a hearing child of Deaf adults (CODA) and highly skilled in working with Deaf people, capturing ASL on film, and creating unifying plots to deliver a large amount of didactic content in an entertaining fashion. Multiple iterations of the script were generated by the research team and filmmaker until consensus was reached on a final version.

Together with the filmmaker, our team recruited a diverse group of Deaf and hearing actors to the project-diverse with respect to race/ethnicity, educational background, and current professions (e.g., elementary school teachers, ASL/Deaf Studies professors, behavioral healthcare workers). Some had experience with professional acting, while others had none. When selecting actors, we were mindful to recruit individuals who were skilled in translating their English lines into ASL-the English script was considered a guide, and each actor applied their own style to the translation to create a greater sense of authenticity.

Film locations were similarly selected for authenticity and included the training and simulation center at the University of Massachusetts Medical School, a formal meeting room at Brown University, and a professional film studio. The film crew was comprised of mostly Deaf or hearing ASL-fluent individuals, and one of our research team's Deaf Community Advisors served in the role of Co-Director. When non-signing crew members were present, ASL interpreters were present to facilitate communication on set. ASL and content consultants were also present on set to provide feedback on ASL translations during the filming process.

Following the completion of filming, our research team was also an integral part of the video editing process. A Deaf artist was hired to produce visual art to augment narration and dialogue throughout the video. The video was designed to be fully ASL/English bilingual so that it can be understood by both signing and non-signing viewers. Subtitles were included throughout for added accessibility. Again, the research team provided feedback on multiple iterations of the training videoDeaf team members focused more on the linguistic and visual aspects of the video, while hearing team members provided particular guidance on sound mixing, music, and voiceover. Of note, there were instances in which the visuals and subtitles were not in sync for Deaf viewers but were for hearing viewers. When this occurred, our team chose to align the experience for hearing viewers, given they were our intended target audience. During the editing process, our team also elicited feedback from hearing research staff to ensure that the video was congruent with hearing culture, easily understandable, and sufficiently sensitive in tone. A selection of screenshots from the final training video 
Table 1 Qualitative themes and subthemes from Deaf community forums

\begin{tabular}{|c|c|}
\hline Themes & Subthemes \\
\hline Need for system change & $\begin{array}{l}\text { IRB practices/policies } \\
\text { NIH/other funder practices/policies } \\
\text { Interpreter practices/policies } \\
\text { National Deaf research resource center }\end{array}$ \\
\hline Information for researcher training intervention & $\begin{array}{l}\text { Training approach/delivery } \\
\text { Training content: } \\
\text { - Why it is important to include Deaf participants in research } \\
\text { - Deaf history, culture, and oppression } \\
\text { - Deaf community diversity } \\
\text { - Visual-manual orientation } \\
\text { - Communication preferences and requirements } \\
\text { - How to request interpreter(s) } \\
\text { - How to prepare interpreter(s) } \\
\text { - How to work with interpreter(s) } \\
\text { - Use of tele-technology } \\
\text { - Emphasizing the importance of the informed consent process to } \\
\text { - participant } \\
\text { - Checking for participant understanding } \\
\text { - Budgeting for accommodations }\end{array}$ \\
\hline Information regarding informed consent form & $\begin{array}{l}\text { Reading ease } \\
\text { Order/structure of content } \\
\text { Formatting } \\
\text { Use of visual aids } \\
\text { Teaching through examples } \\
\text { Explanation of accommodations for inaccessible study procedures } \\
\text { Contact information } \\
\text { Alternate versions of Informed Consent Form }\end{array}$ \\
\hline Information for interpreter training & $\begin{array}{l}\text { Training approach/delivery } \\
\text { Training content: } \\
\text { - Deaf history, culture, and oppression } \\
\text { - Principles of informed consent } \\
\text { - Necessary content knowledge } \\
\text { - Pre-meeting with researcher } \\
\text { - Interpreter role and influence on informed consent process } \\
\text { - Teaching through examples } \\
\text { - Assessing need for CDI }\end{array}$ \\
\hline & \\
\hline
\end{tabular}

is located in Figure 1. The full video can be accessed publicly at https://www.nih.gov/health-information/nih-clinical-researchtrials-you/guidelines-communicating-informed-consentindividuals-who-are-deaf-or-hard-hearing-scientists.

\section{Pilot Study Methods}

The final objective of our 2-year study was to test the feasibility, acceptability, and preliminary efficacy of the training intervention described above by using state-of-the-art medical simulation techniques, outlined in more detail below.

Recruitment. We recruited five research staff members (e.g., research coordinators, research project directors) from the University of Massachusetts Medical School who were currently or recently engaged in conducting informed consent for human subjects research. Recruitment materials advertised a study on "conducting culturally and linguistically appropriate informed consent." Two primary recruitment methods were used: (a) email distribution to faculty and staff of the Department of Psychiatry and (b) paper flyers posted throughout the primary research buildings on the medical school campus (Figure 2).

Inclusion criteria were as follows: (a) age 18+; (b) current or recent engagement in informed consent procedures for human subjects research; and (c) access to an informed consent form that could be used for a simulated informed consent session (so that they could practice conducting culturally appropriate informed consent using content with which they were already familiar).

Procedures and measures. Eligible participants were scheduled for a 4-hr study session with our team in the University of Massachusetts Medical School Interprofessional Center for Experiential Learning and Simulation (iCELS). Medical simulation can be defined as any educational activity that utilizes simulative aides to replicate clinical scenarios (e.g., intravenous insertion arms, resuscitation manikins, standardized patients/participants; Al-Elq, 2010). Medical simulation has been demonstrated to improve performance in technical and clinical skills, critical thinking, communication skills, professionalism, patient care delivery, and resulting patient health outcomes (Beaubien \& 

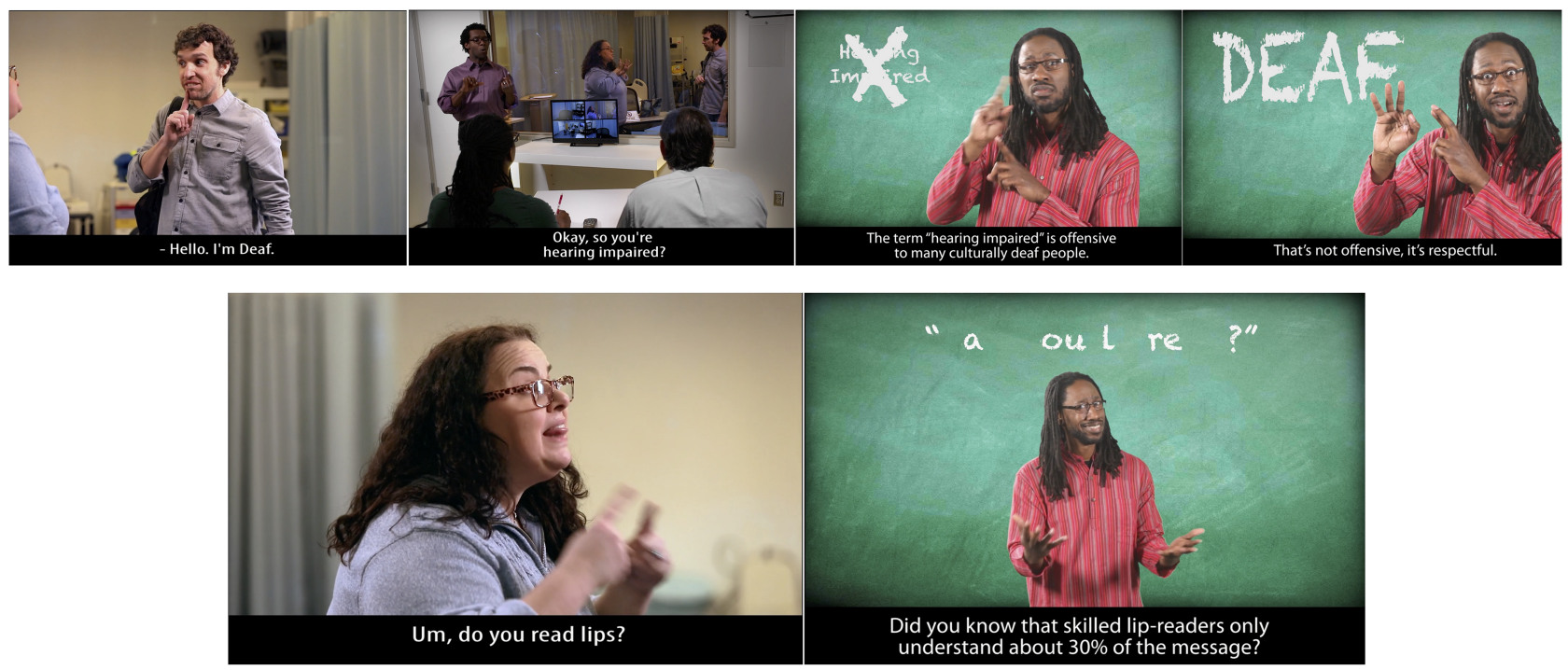

Figure 1 Selection of six screenshots from researcher training video publicly available at https://www.nih.gov/health-information/nih-clinical-research-trials-you/ guidelines-communicating-informed-consent-individuals-who-are-deaf-or-hard-hearing-scientists

\section{Mean Evaluation Scores}

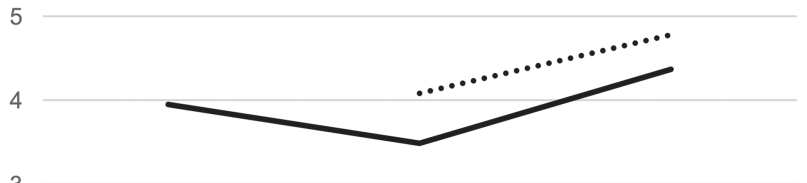

3

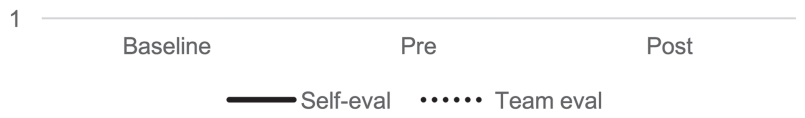

Figure 2 Sample means from participant self-evaluations and research team member evaluations.

Baker, 2004; Cook et al., 2011; Cook et al., 2012; Cook, Brydges, Zendejas, Hamstra, \& Hatala, 2013a, 2013b; Gordon et al., 2010; McGaghie, Draycott, Dunn, Lopez, \& Stefanidis, 2011). In addition, simulation has been used to improve the skills of clinical trials coordinators (Taekman et al., 2004; Wright et al., 2005) and to increase cultural competency of nurses and physicians in training (Aeder et al., 2007; Green et al., 2007; Miller \& Green, 2007; Rutledge et al., 2008; Yang, Woomer, Agbemenu, \& Williams, 2014).

During each study session, the participating research staff member engaged in two simulated encounters in which they were instructed to conduct informed consent procedures with a Deaf standardized participant (i.e., one of our team's Deaf Community Advisors acting the role of a Deaf research participant). Prior to arriving to the study session, participants were not aware that they would be meeting with a Deaf ASL user for their "culturally and linguistically appropriate informed consent" experience. Specific procedures were as follows:

1. Pre-Brief and Informed Consent. The principal investigator provided a detailed overview of the study, conducted informed consent procedures, and obtained written informed consent.
2. Pre-Intervention Evaluation. Participants completed a preintervention evaluation that queried demographic characteristics, professional experience, level of prior experience with the Deaf community, and a self-assessment of perceived informed consent skills at baseline.

3. Simulation \#1. The iCELS lab was arranged to reflect a realworld informed consent setting (i.e., patient room in a teaching hospital). The following tools and resources were placed in the room to facilitate the interaction: a white board with dry erase markers, paper and pens, and a sign with information about how to contact Interpreter Services (which listed a direct phone line to our research team's ASL interpreter). Deaf research team members observed the simulation in real time from the iCELS viewing room. Simulation encounters were videotaped through the LearningSpace system with capacity for playback and review, as needed.

4. Rating of Simulation \#1. Team members who observed or participated in the simulation completed an evaluation immediately after Simulation \#1. This evaluation assessed the participant's performance conducting informed consent prior to the training intervention, prompted suggestions for improvement, and asked if they would be willing to provide consent.

5. Training Video. Next, participants watched Sign Here: How to Conduct Informed Consent with Deaf Research Participants. The video sought to teach viewers about the basics of Deaf history and culture, how to interact with a Deaf research participant through interpreters, and how to design culturally appropriate informed consent forms.

6. Self-Rating of Simulation \#1. After viewing the training video, participants completed another self-assessment of informed consent skills, this time rating their performance during Simulation \#1 and noting possible areas of improvement for the second simulation experience.

7. Simulation \#2. Participants then repeated the simulated informed consent encounter. As in Simulation \#1, the simulation was videotaped and observed in real time by Deaf team members.

8. Rating of Simulation \#2. Team members who observed and who participated in the simulation completed an evaluation immediately after Simulation \#2. 
9. Post-Intervention Evaluation. Participants completed a final self-assessment to rate their performance conducting informed consent in the second simulation, as well as to provide feedback on the training video and simulation experience.

10. Debrief. After evaluations were completed, participants received direct feedback from Deaf team members and the Deaf standardized participant about their performance in the simulations. An emphasis was given on providing positive reinforcement and concrete suggestions for further improvement. Participants received a \$100 American Express Gift Card as compensation.

Analyses. The primary objective of this five-participant pilot test was to examine the feasibility and acceptance of our researcher training intervention. Feasibility outcomes included recruitment, retention, engagement, participant satisfaction, intervention fidelity, and feasibility of assessment procedures. Exploratory efficacy outcomes included differences in selfassessed and research-team-assessed performance ratings before and after the simulation. We used repeating, continuous scale measures (i.e., 5-point Likert scales) that could be analyzed directly via pre-post differences.

\section{Pilot Study Results}

Participants. Participants were four female and one male research staff members, representing a variety of research disciplines (e.g., Emergency Medicine, Preventative Medicine, Child and Adolescent Neurodevelopment). Age ranged from 27 to 62 years (mean $=45.6$ ). Four participants identified as White non-Hispanic/Latino; one as Asian. Two held graduate degrees, while three held 4-year college degrees. Work experience ranged from 3 to 30 years (mean=11.4). None of the participants had participated in simulation-based training prior to the current study. Two participants reporting receiving 1-3 hr of formal training in conducting informed consent procedures; two reported 4-10 hr of training; and one reported 11-40 hr of training. Four participants reported $40+\mathrm{hr}$ of hands-on experience conducting informed consent; one reported 11-40 hr of experience. Two participants reported "a lot" of experience interacting with Deaf individuals (i.e., I have met more than a few Deaf people and have had meaningful interactions with them), while the other three reported "very little" (i.e., I have met one or two Deaf people but did not have meaningful interactions with them).

Feasibility and acceptability. Across 10 weeks of open recruitment, a total of 29 individuals contacted our research team to participate in the current pilot study, which resulted in the five study slots being filled within the first week of recruitment. This level of interest was generated only with paper flyers posted at our university's main campus and one recruitment email sent to the Department of Psychiatry. Three out of five of the originally scheduled participants who were originally scheduled completed the study; two original participants had to cancel their study sessions due to other obligations but were successfully replaced with other eligible individuals who had been placed on the study waitlist.

Regarding participant engagement, satisfaction, and the acceptability of our simulation-based training intervention, all five participants "strongly agreed" (i.e., 5 on a 5-point Likert scale) that the researcher training video was helpful and that the simulation-based practice was helpful. Four participants "strongly agreed" that they felt more confident implementing culturally appropriate informed consent as a result of their training session; one participant "agreed" with this statement (i.e., 4 on a 5-point Likert scale). A selection of qualitative comments regarding acceptability of the researcher training is noted in Table 2.

Preliminary efficacy outcomes. As can be seen in Table 3, each participant reported a perceived improvement in performance from pre- to post-intervention, with the overall sample mean increasing nearly one point on a 5 -point Likert scale $(1=$ not well at all, 3 =somewhat well, $5=$ very well). Although participants generally rated themselves more critically than did members of the research team, team member ratings corroborated participants' reported improvement, with the magnitude of change scores very similar across self-ratings and team member ratings. Participants who reported that they had "very little" prior experience interacting with Deaf individuals made greater gains in their post-intervention performance than those who had "a lot" of experience. This finding confirms that the appropriate target audience for the developed training intervention is hearing research staff with little-to-no prior exposure to the Deaf community.

Of note, following the first simulation experience and training video, four out of five participants reported a reduction in their perceived ability to conduct culturally appropriate informed consent from baseline (i.e., before the first simulated informed consent session). This suggests that the initial simulation may have challenged their preexisting confidence in their own cultural competency and/or that the training video content provided a better context to evaluate one's ability to conduct culturally competent informed consent procedures.

\section{Discussion}

Our team's three-phase, community-engaged, formative study culminated in a training video and simulation intervention that can be feasibly implemented with hearing researchers to improve their conduct of culturally and linguistically appropriate informed consent with Deaf research participants. Results of our small, five-participant pilot study supported the acceptability and feasibility of our developed training intervention for future research endeavors and indicated consistent improvement in participants' ability to conduct informed consent-as perceived by both the participants themselves and research team members who observed or participated in the simulated informed consent encounters.

In addition to the tangible contribution that this training intervention will make to the field, the current study represents a significant leap forward for the field of Deaf health research. The current study is the first-ever application of an evidencebased, community-engaged approach to adapt informed consent within the Deaf population and is notable for hiring four Deaf Community Advisors to play a lead role in developing and delivering the training intervention. Second, our approach was unique in the application of the Truth and Reconciliation Model during the Phase 1 Deaf Community Forums, an emerging strategy to promote engagement between researchers and minority community members (Twombley, 2014). Third, training research staff members in a state-of-the-art simulation environment with a Deaf standardized participant provided a relatively novel basis for our training intervention.

Despite these innovations, the current study also possesses limitations. As noted in the Deaf Community Forum results, community member turnout was much lower than we had 
Table 2 Qualitative responses regarding pilot participant engagement, satisfaction, and intervention acceptability

\begin{tabular}{|c|c|}
\hline Evaluation question & Selected qualitative responses \\
\hline $\begin{array}{l}\text { 1. How has participation in Deaf ACCESS changed the way you } \\
\text { implement informed consent? }\end{array}$ & $\begin{array}{l}\text { - I learned some very valuable information about working with Deaf } \\
\text { participants that I believe will make me ask myself if there is any way } \\
\text { I can be more respectful of folks from other populations as well. } \\
\text { - It forced me to go through each section more thoroughly where I took } \\
\text { my time. Typically the consent process is much quicker but with this } \\
\text { practice I felt confident the subject understood the study. } \\
\text { - I think it will help me format my informed consents with using visual } \\
\text { information more effectively. Also, cueing into jargon and acronyms } \\
\text { more carefully with all participants. }\end{array}$ \\
\hline 2. Was the time allocation appropriate on the training video? & $\begin{array}{l}\text { - Yes. I think the training video conveyed a significant range of issues to } \\
\text { be considered in a succinct way. The video was an effective vehicle to } \\
\text { do this. }\end{array}$ \\
\hline 3. Was the content relevant on the training video? & $\begin{array}{l}\text { - Yes. A lot of times assumptions are made in the Emergency } \\
\text { Department as to how Deaf patients want to communicate. }\end{array}$ \\
\hline 4. What did you like best about the training video? & $\begin{array}{l}\text { - I think the video was challenging in a positive way. It has started me } \\
\text { to think about how to better explain research events to participants. } \\
\text { I did not know there was more than } 2 \text { ways to communicate with } \\
\text { Deaf patients. Very educational. } \\
\text { - I liked the jaunty, friendly feel of the video. I like that you gently } \\
\text { explained why it is not ok to ask folks if they lipread. }\end{array}$ \\
\hline $\begin{array}{l}\text { 5. What did you like best about the experience of the simulation } \\
\text { exercise? }\end{array}$ & $\begin{array}{l}\text { - The "in the moment" thinking and responding to the potential } \\
\text { participant, with the benefit of getting feedback about my responses. } \\
\text { This will help me do a better job of consenting. "Real" participants } \\
\text { typically do not give that kind of feedback. } \\
\text { - I learnt new things today about improving my competency in working } \\
\text { with Deaf patients. I feel more confident now. ASL need not be seen as } \\
\text { a communication barrier between patient and clinician. }\end{array}$ \\
\hline
\end{tabular}

Table 3 Mean scores of participant self-evaluations and research team member evaluations

\begin{tabular}{|c|c|c|c|c|c|c|c|}
\hline & \multicolumn{4}{|c|}{ Participant self-evaluation scores } & \multicolumn{3}{|c|}{ Research Team Evaluation Scores } \\
\hline & Baseline $^{\mathrm{a}}$ & $\begin{array}{c}\text { Pre- } \\
\text { intervention }^{\mathrm{b}}\end{array}$ & $\begin{array}{c}\text { Post- } \\
\text { intervention }\end{array}$ & $\begin{array}{l}\text { Difference } \\
\text { (post-pre) }\end{array}$ & $\begin{array}{c}\text { Pre- } \\
\text { intervention }^{\mathrm{b}}\end{array}$ & $\begin{array}{c}\text { Post- } \\
\text { intervention }\end{array}$ & $\begin{array}{l}\text { Difference } \\
\text { (post-pre) }\end{array}$ \\
\hline & 3.18 & 4.00 & 4.36 & .36 & 4.53 & 4.65 & .12 \\
\hline & 3.73 & 3.45 & 3.99 & .54 & 4.13 & 4.76 & .63 \\
\hline & 4.65 & 3.18 & 4.95 & 1.77 & 3.27 & 4.54 & 1.26 \\
\hline & 3.81 & 3.36 & 3.99 & .63 & 4.57 & 5 & .43 \\
\hline & 4.36 & 3.45 & 4.54 & 1.09 & 3.89 & 4.95 & 1.06 \\
\hline Sample mean & 3.95 & 3.49 & 4.37 & .88 & 4.08 & 4.78 & .70 \\
\hline
\end{tabular}

${ }^{a}$ Baseline evaluation occurred before the first simulation experience.

$\mathrm{b}$ Pre-intervention evaluation occurred after the first simulation experience, but before the training video.

originally anticipated, likely due to deep-seeded mistrust of researchers and/or limited understanding of the meaning and potential benefits of research participation. As we come to the end of our 2-year, community-engaged study, we hope that some of this mistrust and misunderstanding has begun to subside. This expectation will be tested at an upcoming "red carpet movie premiere" of our training video, specifically hosted to show appreciation to local Deaf community members and community partners for their support, to keep them apprised of study results, and inform them of next steps.

With regard to next steps, one of our primary goals is to replicate the pilot study methods described above within a large sample of researchers and research staff members, across a number of biomedical disciplines and universities.
This expansion of our study sample will allow us to make more confident conclusions about the efficacy of our developed training intervention, examine the relative efficacy of different levels or components of the intervention (i.e., training video alone, training video plus simulation-based practice, training video plus simulation-based practice, and post-simulation debriefing), and explore the generalizability of our intervention across academic settings with varied training resources.

If evidence of efficacy is found, widespread implementation and dissemination of the Deaf ACCESS training intervention-for example, via the Research Ethics and Compliance Training provide by the Collaborative Institutional Training Initiative (CITI) Program-has the potential to prompt a paradigm shift in how 
biomedical researchers conduct informed consent in the Deaf community. Such a shift could increase the number of Deaf people willing and able to participate in research and, more importantly, move from Deaf people as research participants to Deaf people as investigators actively engaged in and leading biomedical research.

\section{Funding}

This work was supported by the National Institute on Deafness and Other Communication Disorders (NIDCD) of the National Institutes of Health (Award Number R21DC015580). The content is solely the responsibility of the creators and does not necessarily represent the official views of the National Institutes of Health.

\section{Conflicts of Interest}

No conflicts of interest were reported.

\section{References}

Aeder, L., Altshuler, L., Kachur, E., Barrett, S., Hilfer, A., Koepfer, S., \& Shelov, S. P. (2007). The "culture OSCE"-Introducing a formative assessment into a postgraduate program. Education for Health (Abingdon, England), 20(1), 11.

Al-Elq, A. H. (2010). Simulation-based medical teaching and learning. Journal of Family and Community Medicine, 17(1), 35-40. doi: 10.4103/1319-1683.68787

Anderson, M. L., Riker, T., Gagne, K., Hakulin, S., Higgins, T., Meehan, J., \& Wolf Craig, K. S. (2018). Deaf qualitative Health Research: Leveraging technology to conduct linguistically and Sociopolitically appropriate methods of inquiry. Qualitative Health Research, 28(11), 1813-1824. doi: $10.1177 / 1049732318779050$

Anderson, M. L., Ziedonis, D. M., \& Najavits, L. M. (2014). Posttraumatic stress disorder and substance use disorder comorbidity among individuals with physical disabilities: Findings from the national comorbidity survey replication. Journal of Traumatic Stress, 27(2), 182-191. doi: 10.1002/jts.21894

Barnett, S., Klein, J. D., Pollard, R. Q. Jr., Samar, V., Schlehofer, D., Starr, M., \& Pearson, T. A. (2011). Community participatory research with deaf sign language users to identify health inequities. American Journal of Public Health, 101(12), 2235-2238. doi: 10.2105/AJPH.2011.300247

Barnett, S., McKee, M., Smith, S. R., \& Pearson, T. A. (2011). Deaf sign language users, health inequities, and public health: Opportunity for social justice. Preventing Chronic Disease, 8(2), A45.

Bauman, H. D. (2004). Audism: Exploring the metaphysics of oppression. Journal of Deaf Studies and Deaf Education, 9(2), 239-246. doi: 10.1093/deafed/enh025

Beaubien, J. M., \& Baker, D. P. (2004). The use of simulation for training teamwork skills in health care: How low can you go? Quality \& Safety in Health Care, 13(Suppl 1), i51-i56. doi: 10.1136/qhc.13.suppl_1.i51

Cook, D. A., Brydges, R., Hamstra, S. J., Zendejas, B., Szostek, J. H., Wang, A. T., \& Hatala, R. (2012). Comparative effectiveness of technology-enhanced simulation versus other instructional methods: A systematic review and meta-analysis. Simulation in Healthcare, 7(5), 308-320. doi: 10.1097/SIH.0b013e3182614f95
Cook, D. A., Brydges, R., Zendejas, B., Hamstra, S. J., \& Hatala, R. (2013a). Mastery learning for health professionals using technology-enhanced simulation: A systematic review and meta-analysis. Academic Medicine, 88(8), 1178-1186. doi: 10.1097/ACM.0b013e31829a365d

Cook, D. A., Brydges, R., Zendejas, B., Hamstra, S. J., \& Hatala, R. (2013b). Technology-enhanced simulation to assess health professionals: A systematic review of validity evidence, research methods, and reporting quality. Academic Medicine, 88(6), 872-883. doi: 10.1097/ACM.0b013e31828ffdcf

Cook, D. A., Hatala, R., Brydges, R., Zendejas, B., Szostek, J. H., Wang, A. T., \& Hamstra, S. J. (2011). Technology-enhanced simulation for health professions education: A systematic review and meta-analysis. JAMA, 306(9), 978-988. doi: 10.1001/jama.2011.1234

Fellinger, J., Holzinger, D., \& Pollard, R. Q. (2012). Mental health of deaf people. Lancet, 379(9820), 1037-1044. doi: 10.1016/S0140-6736(11)61143-4

Glickman, N. S. (2013). Lessons learned from 23 years on a deaf psychiatric inpatient unit. In N. S. Glickman (Ed.), Deaf mental health care (pp. 37-68). New York, NY: Routledge.

Gordon, J. A., Hayden, E. M., Ahmed, R. A., Pawlowski, J. B., Khoury, K. N., \& Oriol, N. E. (2010). Early bedside care during preclinical medical education: Can technology-enhanced patient simulation advance the Flexnerian ideal? Academic Medicine, 85(2), 370-377. doi: 10.1097/ACM.0b013e3181c88d74

Green, A. R., Miller, E., Krupat, E., White, A., Taylor, W. C., Hirsh, D. A., \& Betancourt, J. R. (2007). Designing and implementing a cultural competence OSCE: Lessons learned from interviews with medical students. Ethnicity \& Disease, 17(2), 344-350.

Krueger, R. A. (1998). Analyzing and reporting focus group results (). Thousand Oaks, CA: Sage Publications.

Ladd, P. (2003). Understanding deaf culture: In search of deafhood (). Tonawanda, NY: Multilingual Matters.

Lane, H. (1992). The mask of benevolence: Disabling the deaf community. New York, NY: Alfred A. Knopf, Inc.

Lane, H. (2005). Ethnicity, ethics, and the deaf-world. Journal of Deaf Studies and Deaf Education, 10(3), 291-310. doi: 10.1093/deafed/eni030

Livermore, G., Whalen, D., Prenovitz, S., Aggarwal, R., \& Bardos, M. (2011). Disability data in national surveys: Prepared for the Office of Disability, Aging and Long-Term Care PolicyRetrieved from. https://aspe.hhs.gov/basic-report/disability-datanational-surveys.

McEwen, E., \& Anton-Culver, H. (1988). The medical communication of deaf patients. The Journal of Family Practice, 26(3), 289-291.

McGaghie, W. C., Draycott, T. J., Dunn, W. F., Lopez, C. M., \& Stefanidis, D. (2011). Evaluating the impact of simulation on translational patient outcomes. Simulation in Healthcare, 6(Suppl), S42-S47. doi: 10.1097/SIH.0b013e318222fde9

McKee, M., Schlehofer, D., \& Thew, D. (2013). Ethical issues in conducting research with deaf populations. American Journal of Public Health, 103(12), 2174-2178. doi: 10.2105/AJPH.2013.301343

Miller, E., \& Green, A. R. (2007). Student reflections on learning cross-cultural skills through a 'cultural competence' OSCE. Medical Teacher, 29(4), e76-e84. doi: 10.1080/01421590701266701

Mitchell, R., Young, T., Bachleda, B., \& Karchmer, M. (2006). How many people use ASL in the United States? Why estimates need updating. Sign Language Studies, 6, 306-335. doi: $10.1353 /$ sls.2006.0019 
Morere, D. A. (2011). Reading research and deaf children. Visual Language \& Visual Learning Research Brief, 4. http://vl2.gallaudet.edu/files/8713/9216/6286/researchbrief-4-reading-and-deaf-children.pdf

Rutledge, C. M., Barham, P., Wiles, L., Benjamin, R. S., Eaton, P., \& Palmer, K. (2008). Integrative simulation: A novel approach to educating culturally competent nurses. Contemporary Nurse, 28(1-2), 119-128. doi: 10.5172/conu.673.28.1-2.119

Taekman, J. M., Hobbs, G., Barber, L., Phillips-Bute, B. G., Wright, M. C., Newman, M. F., \& Stafford-Smith, M. (2004). Preliminary report on the use of high-fidelity simulation in the training of study coordinators conducting a clinical research protocol. Anesthesia and Analgesia, 99(2), 521-527. doi: 10.1213/01.ANE.0000132694.77191.BA

Twombley, R. (2014). To reduce health disparities, first acknowledge past research wrongs. Georgetown University Medical Center News. Retrieved from
http://gumc.georgetown.edu/news/To-Reduce-HealthDisparities-First-Acknowledge-Past-Research-Wrongs

Wong, J. P., \& Poon, M. K. (2010). Bringing translation out of the shadows: Translation as an issue of methodological significance in cross-cultural qualitative research. Journal of Transcultural Nursing, 21(2), 151-158. doi: 10.1177/1043659609357637

Wright, M. C., Taekman, J. M., Barber, L., Hobbs, G., Newman, M. F., \& Stafford-Smith, M. (2005). The use of high-fidelity human patient simulation as an evaluative tool in the development of clinical research protocols and procedures. Contemporary Clinical Trials, 26(6), 646-659. doi: 10.1016/j.cct.2005.09.004

Yang, K., Woomer, G. R., Agbemenu, K., \& Williams, L. (2014). Relate better and judge less: Poverty simulation promoting culturally competent care in community health nursing. Nurse Education in Practice, 14(6), 680-685. doi: 10.1016/j.nepr.2014.09.001 